\title{
Über meiotische Degenerationsvorgänge bei polyploiden Tomaten
}

\author{
Werner Gottschalk \\ Institut für Genetik der Universität Bonn, Bonn, Deutschland
}

Eingegangen am 22. September 1965

\section{A. Einleitung}

Uber pathologische Vorgänge während des mitotischen und meiotischen Stadienablaufs liegt eine umfangreiche Literatur vor (Tischler 1951, Tischler und Wulff 1953-63). Die Anomalien können sowohl spontan als auch nach Anwendung der verschiedenartigsten physikalischen und chemischen Agentien auftreten. Trotz der Vielfalt des Gesamtgeschehens ist das Spektrum relativ schmal, wenn wir die Befunde an bestimmten Einzelobjekten bzw. die Wirkung eines einzigen spezifischen Agens beurteilen. Im Rahmen ausgedehnter cytologischer Studien an experimentell erzeugten polyploiden Tomaten mit Valenzen von 4-8n war es möglich, eine große Anzahl meiotischer und prämeiotischer Regulierungsmodi nachzuweisen. Sie sind in karyologischer Beziehung als Modifikanten der Meiosis mit eigenen Verteilungsgesetzen aufzufassen und führen zu Keimzellen mit der mehrfach reduzierten Chromosomenzahl. Als Regulierungssysteme stellen sie die cytologischen Mechanismen für das seit langem bekannte Phänomen der Rückregulierung der Chromosomenzahl vom polyploiden zum diplo- oder haploiden Zustand dar (Einzelheiten bei Gottschalk 1958, 1959, 1960, Gottschalk und Heide 1960).

Das besondere Kennzeichen derartiger polyploider Versuchspflanzen besteht in einer hochgradigen cytologischen Labilität, die sich nicht nur in Form der eben erwähnten Regulierungsvorgänge, sondern darüberhinaus noch in Form einer Fülle verschiedenartigster meiotischer Unregelmäßigkeiten äußert. Sie sind teils pathologischer Natur, teilweise stehen sie jedoch in einer engen Beziehung zum genomatischen Regulierungsgeschehen und gewinnen dadurch an Interesse. In der vorliegenden Arbeit sollen aus dem breiten Spek. trum derartiger Störungstypen die Degenerationsprozesse sowie die Spindelanomalien behandelt werden.

\section{B. Material und Methode}

Es wurden diploide und tetraploide Samen der Handelssorten "Immun" und "Bonner Beste" von Lycopersicon esculentum auf Colchicin-Agar nach Swaminathan (1950) angekeimt. Unter den wenigen überlebenden Pfianzen befanden sich neben tetraploiden auch einige höherpolyploide Individuen mit sehr labilen cytologischen Verhältnissen. Am gesamten Material wurde der meiotische Stadienablauf analysiert. Hierzu wurden die Antheren junger Blütenknospen in Carnoy 3:1 fixiert, mit Eisessigkarmin unter Eisenzusatz gefärbt und in Form von Quetschpräparaten verarbeitet.

\section{Die empirischen Befunde}

1. Die Degeneration von Chromosomen in der Meiosis

In unserem Material wurden sowohl Verklebungen als auch Degenerationserscheinungen vielfältiger Art beobachtet, wobei eine Trennung dieser beiden Anomalien nicht in allen Fällen mëglich war. Die Verklebung von 
Chromosomen ist eine weit verbreitete Teilungsanomalie, die als pathologische Erscheinung nach Einwirkung von Röntgenstrahlen und mutagenen Chemikalien regelmäßig auftritt und auf Matrixschädigungen zurückgeführt wird. In den Pollenmutterzellen (PMZ) unserer polyploiden Pflanzen ging die Anomalie teilweise so weit, da $B$ sich alle im Kern vorhandenen Chromosomen zu einer amorphen, strukturlosen Chromatinmasse vereinigten, die offenbar keine normale Funktion mehr übernehmen kann. Das meiotische Teilungsstadium derartiger Zellen konnte nur indirekt aus dem Verhalten nicht geschädigter Nachbarzellen erschlossen werden. In der Mehrzahl aller Fälle war die Störung in den Stadien Meta- bis Telophase beider Teilungen zu beobachten; die der Anomalie zugrunde liegenden Ursachen dürften also auch hier über Matrixschädigungen wirksam werden.

Häufig sind beide Anomalien im gleichen Kern realisiert. Eine derartige Zelle ist in Abb. 1 wiedergegeben. Hier tritt die Verklebung nur als Teileffekt einer allgemeinen degenerativen Veränderung des Gonotokonten in Erscheinung. Schon die Grundstruktur des Kerns ist mit keinem Stadium des meiotischen Normalverhaltens vergleichbar. Darüberhinaus sind große, schollige Chromatinmassen erkennbar, die nur durch Verklebung eines Teils des chromatischen Materials zustande gekommen sein können. Der Effekt trat übereinstimmend in mehr als $50 \mathrm{PMZ}$ der gleichen Antherenregion der oktoploiden Pflanze Nr. 15 auf; die Nachbarzellen durchliefen die Diakinese.

In Ausnahmefällen führen degenerative Vorgänge zur Bildung von Kernen, die in ganz extremer Weise vom meiotischen Normalzustand abweichen und keine Deutung mehr gestatten. So ist die in Abb. 2 dargestellte PMZ einerseits durch ihr anomal großes Zellvolumen, anderseits durch ein stark reduziertes Kernvolumen charakterisiert. Ihr kugelförmiger Kern ist von einer Membran umschlossen; im Inneren sind chromosomenähnliche Gebilde, vielleicht auch Nukleolen, erkennbar, es ist jedoch nicht möglich, nähere Aussagen über die Kernstruktur zu machen. Dieser Störungstypus trat übereinstimmend innerhalb kleiner, eng begrenzter Bezirke verschiedener Antheren der tetraploiden Pflanze Nr. 599 auf.

Die degenerativen Veränderungen der Chromosomen können so weit gehen, daß sich das gesamte im Kern vorhandene Chromosomenmaterial auflöst. Wir haben mehrfach in der gleichen Antherenregion abnormale Kerne gefunden, die in eine abgestufte Reihe zunehmenden degenerativen Verfalls eingeordnet werden können. Der Effekt ist im einzelnen nicht analysierbar, es hat jedoch den Anschein, als würde die bereits weit fortgeschrittene Spiralisation der Chromosomen rückgängig gemacht. Im weiteren Verlauf des Prozesses verschwindet der Unterschied zwischen Hetero- und Euchromatin immer mehr, bis schlieBlich ein Zustand erreicht wird, der morphologisch etwa der fixierungslabilen Synizesis der meiotischen Prophase entspricht (Abb. 3, 4). Die gesamte Anthere war einwandfrei fixiert; die umliegenden Zellen durchliefen die Stadien MI bis Interkinese und gaben 
saubere cytologische Bilder mit einem für tetraploide PMZ normalen Verhalten. Das Endstadium derartiger Vorgänge besteht offenbar in der Resorption des Chromatins durch das Cytoplasma. In Antheren, die die letzten Stadien der Meiosis enthielten, waren nicht selten ganze Gruppen völlig kernloser Zellen vorhanden.

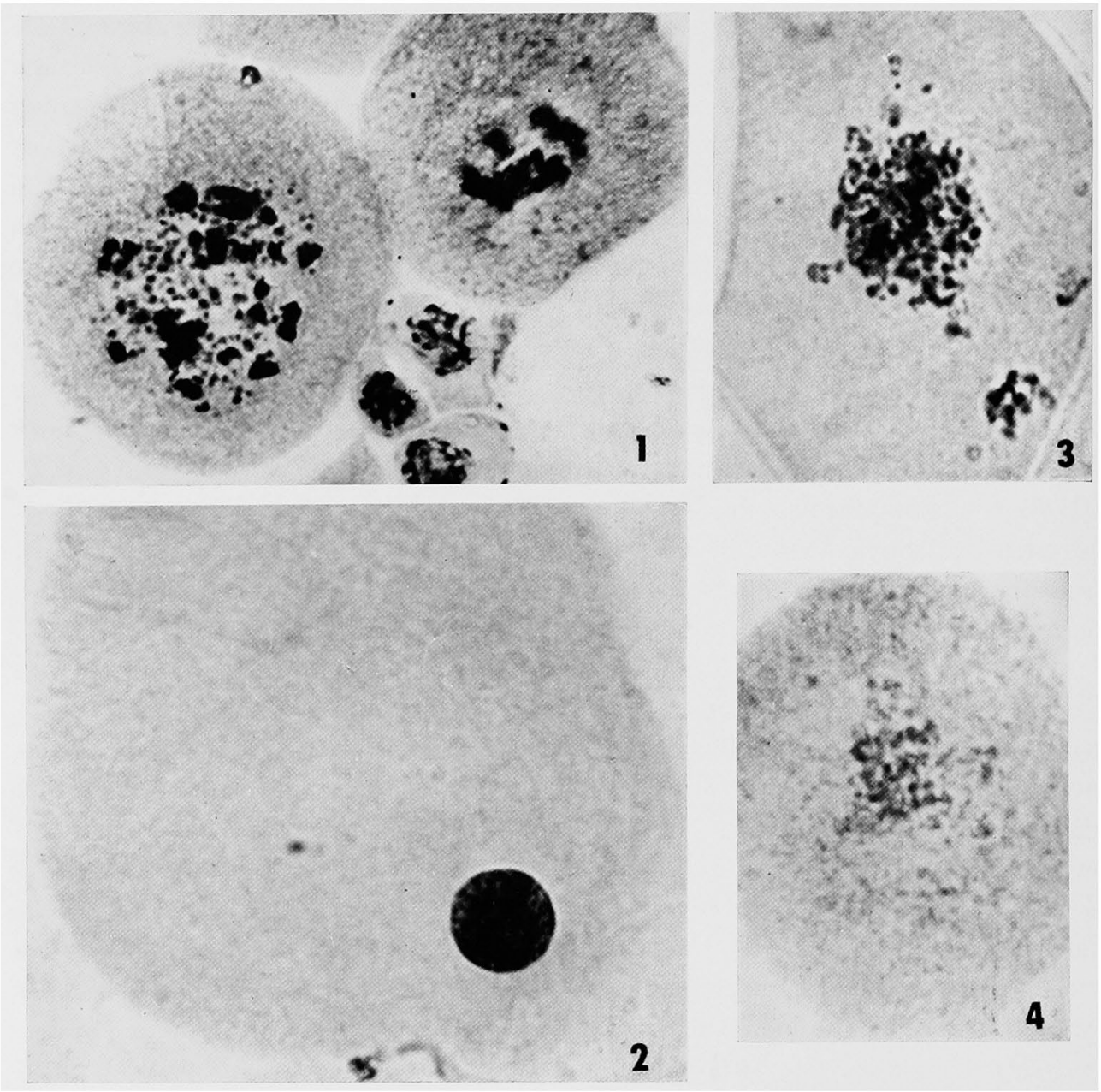

Abb. 1-4. 1, degenerierender meiotischer Kern der oktoploiden Pflanze Nr. 15 mit groben Verklebungen von Chromatinsubstanz. 2, anomal kleiner, atypischer Kern einer großvolumigen Pollenmutterzelle der tetraploiden Versuchspflanze 599. 3 und 4, zwei Stadien einer abgestuften Reihe des degenerativen Verfalls aller im Kern vorhandenen Chromosomen. Die beiden Zellen stammen aus einer eng begrenzten Zone der gleichen Anthere der Pflanze Nr. 599, die ausschließlich degenerierende PMZ enthielt; die Nachbarzellen durchliefen die Stadien MI bis Interkinese.

Häufig wird nur ein Teil der in der Zelle vorhandenen Chromosomen von der pathologischen Veränderung betroffen. Derartige partiellen Degenerationsvorgänge gewinnen an Interesse, wenn wir die genomatischen Verhältnisse der betreffenden PMZ für ihre Interpretation berücksichtigen. In Tabelle 1 sind 20 genomatisch analysierbare Zellen aus verschiedenen Region 
der abregulierenden Versuchspflanze Nr. 934 zusammengestellt, deren Valenz in der Größenordnung von $4 \mathrm{n}$ lag und die sich durch vielfältige meiotische Unregelmäßigkeiten auszeichnete.

Die Tabelle wird nur verständlich, wenn wir die komplizierten Gesetzmäßigkeiten meiotischer und prämeiotischer Abregulierungsvorgänge berücksichtigen, die in einer Serie früherer Arbeiten für unser polyploides Versuchsmaterial nachgewiesen wurden (Gottschalk 1958a, b, 1959, 1960, Gottschalk und Heide 1960). Das gemeinsame Kennzeichen aller in der

Tabelle 1. Die genomatischen Verhältnisse von 20 aneuploiden Pollenmutterzellen mit partiellen Degenerationserscheinungen (Erläuterung im Text)

\begin{tabular}{|c|c|c|c|c|c|}
\hline \multirow{2}{*}{$\begin{array}{l}\text { Nummer } \\
\text { der } \\
\text { PMZ }\end{array}$} & \multirow{2}{*}{$\begin{array}{l}\text { Meiotisches } \\
\text { Stadium }\end{array}$} & \multirow{2}{*}{$\begin{array}{l}\text { Kerne mit ausgewo- } \\
\text { genen genomatischen } \\
\text { Verhältnissen }\end{array}$} & \multicolumn{3}{|c|}{$\begin{array}{c}\text { Uberzählige } \\
\text { Chromosomen }\end{array}$} \\
\hline & & & $\begin{array}{l}\text { ver- } \\
\text { klebt }\end{array}$ & $\begin{array}{l}\text { dege- } \\
\text { nerie- } \\
\text { rend }\end{array}$ & $\begin{array}{l}\text { pachytän- } \\
\text { artig }\end{array}$ \\
\hline 1 & Diakinese & $4 n$ & - & - & + \\
\hline 2 & Prometaphase & $\mathrm{n}$ & - & + & - \\
\hline 3 & 1. Metaphase & $\mathrm{n}$ & - & + & - \\
\hline 4 & 1. Metaphase & $2 n$ & + & - & - \\
\hline 5 & 1. Metaphase & $2 n$ & - & - & + \\
\hline 6 & 1. Anaphase & $4 n$ & - & - & + \\
\hline 7 & 1. Anaphase & $n+n$ & + & - & - \\
\hline 8 & 1. Ana-/Telophase & $4 n+4 n$ & - & - & + \\
\hline 9 & 1. Interphase & $2 n+2 n$ & - & + & - \\
\hline 10 & 1. Interphase & $4 n$ & - & + & - \\
\hline 11 & 1. Interphase & $2 n+n+n$ & + & - & - \\
\hline 12 & 2. Metaphase & $2 n$ & + & - & - \\
\hline 13 & 2. Metaphase & $2 \mathrm{n}$ & - & + & - \\
\hline 14 & 2. Metaphase & $2 n+2 n$ & + & - & - \\
\hline 15 & 2. Anaphase & $2 n+2 n+2 n+2 n$ & - & - & + \\
\hline 16 & 2. Telo-/Interphase & $2 n+2 n$ & - & + & - \\
\hline 17 & 2. Telo-/Interphase & $2 n+2 n+2 n$ & - & + & - \\
\hline 18 & 2. Telo-Interphase & $4 n+2 n+2 n$ & - & + & - \\
\hline 19 & 2. Telo-Interphase & $2 n+2 n+2 n+2 n$ & + & - & - \\
\hline 20 & 2. Telo-/Interphase & $2 n+2 n+n+n$ & - & + & - \\
\hline
\end{tabular}

Tabelle aufgeführten PMZ besteht in der aneuploiden Chromosomenzahl. Trotz des genomatisch nicht balancierten Ausgangszustands werden in diesen Zellen euploide Gonen gebildet. Durch einen Regulierungsvorgang, dessen Mechanismus wir noch nicht kennen, werden die überzähligen Chromosomen von den genomatisch ausgewogenen Chromosomengruppen der PMZ abgesondert. Im weiteren Verlauf der Reduktionsteilung sind sie den meiotischen Verteilungsgesetzen offenbar nicht mehr unterworfen und werden inaktiviert oder eliminiert. Meist liegen sie an der Peripherie der Zelle und stellen teils verklebte, teils degenerierende Chromatinmassen dar. Besonders häufig 
liegen sie jedoch in einem pachytän-artigen Zustand vor. Abbildung 5 enthält eine schwach hypertetraploide Zelle, die das Stadium der Diakinese durchäuft. Im Kern sind zunächst 48 Chromosomen in Form normaler Bi- und Multivalente vorhanden. Die wenigen überzähligen Chromosomen liegen außerhalb des Kerns in weitgehend entspiralisierter Form und nehmen nicht am meiotischen Geschehen teil. In Abb. 6 ist prinzipiell die gleiche Situation für ein etwas späteres Stadium wiedergegeben. Analoge Verhältnisse wurden in PMZ beobachtet, die alle anderen meiotischen Stadien bis zur 2. Interphase durchliefen.

Zwischen den letztgenannten beiden Störungsformen-der degenerativen Auflösung und dem kaum spiralisierten Zustand der überzähligen Chromosomen-lassen sich gleitende Übergänge erfassen, so daß angenommen werden kann, dass wir nur graduelle Verschiedenheiten der gleichen Anomalie vor uns haben. Wahrscheinlich kommt bis zum Ende der Meiosis bei fortschreitender Degeneration eine weitgehende oder völlige Auflösung dieser Chromosomen zustande.

Zum Verständnis der Tabelle seien noch folgende Hinweise gegeben: Die Zellen 1, 6 14, 15 und 19 sind hypertetraploid und zeigen-abgesehen von der Aussonderung der überzähligen Chromosomen-ein normales meiotisches Verhalten. Das gleiche gilt für die hyperoktoploide Zelle Nr. 8. Die PMZ Nr. 4 und 5 sind hyperdiploid, die Zellen 2, 3, 7 hyperhaploid; als Ursachen der niedrigen Chromosomenzahlen sind Abregulierungsvorgänge im Verlauf der prämeiotischen Mitosen anzunehmen. Bei der hyperdiploiden Zelle Nr. 10 ist ein Restitutionskern gebildet worden; analoge Verhältnisse liegen bei den hyferhaploiden Zellen 12, 13, 16 vor. Die genomatischen Verhältnisse der Zelle Nr. 18 werden ebenfalls als Folgen einer Restitutionskernbildung verständlich, die sich jedoch nur im Bereich eines der beiden Kerne der MII abgespielt hat. Die Verteilung von $2 n+n+n$ in der Interkinese der hypertetraploiden PMZ 11 ist in Verbindung mit einer tripolaren Spindel zustandegekommen. Die Zellen Nr. 17 und 20 schließlich sind infolge prämeiotischer Abregulierungsvorgänge hypertriploid. Ihre Chromosomenverteilung während der Meiosis ist jedoch nicht nach zahlenmäßigen, sondern nach genomatischen Gesichtspunkten abgelaufen und hat zur Bildung von Gonen mit euploiden Chromosomenzahlen geführt.

2. Spindelanomalien und andere UnregelmäBigkeiten

Neben den eben behandelten Degenerationserscheinungen waren in unserem Material noch vielfältige Spindelanomalien zu beobachten, von denen nur einige erwähnt werden sollen. AuBer tetrapolaren traten besonders häufig tripolare Spindeln auf (Abb. 7), die in einem unmittelbaren Zusammenhang zu genomatischen Regulierungsprozessen stehen und bereits in einer früheren Publikation berücksichtigt wurden (Gottschalk und Heide 1960). Häufig unterbleibt die Spindelbildung ganz und es entstehen Dyaden bzw. Monaden. Derartige Verhältnisse scheinen auch in der in Abb. 8 wiedergegebenen PMZ der oktoploiden Versuchspflanze Nr. 1 vorzuliegen. Sie durchläuft eindeutig die späten Stadien der 2. meiotischen Teilung; der Zustand der Chromosomen entspricht der 2. Ana- oder Telophase. In der Zelle sind jodoch keine Anzeichen einer Polarität erkennbar, die auf die Funktion von Spindeln hinweisen würden. Dei Zelle besitzt etwa 160 Chro- 
mosomen, die offenbar zu einem einzigen Restitutionskern vereinigt werden. Es entsteht auf diese Weise eine Gone mit der Valenz von annähernd $14 \mathrm{n}$. Analoge Vorgänge laufen nicht nur in der 1. meiotischen Teilung, sondern

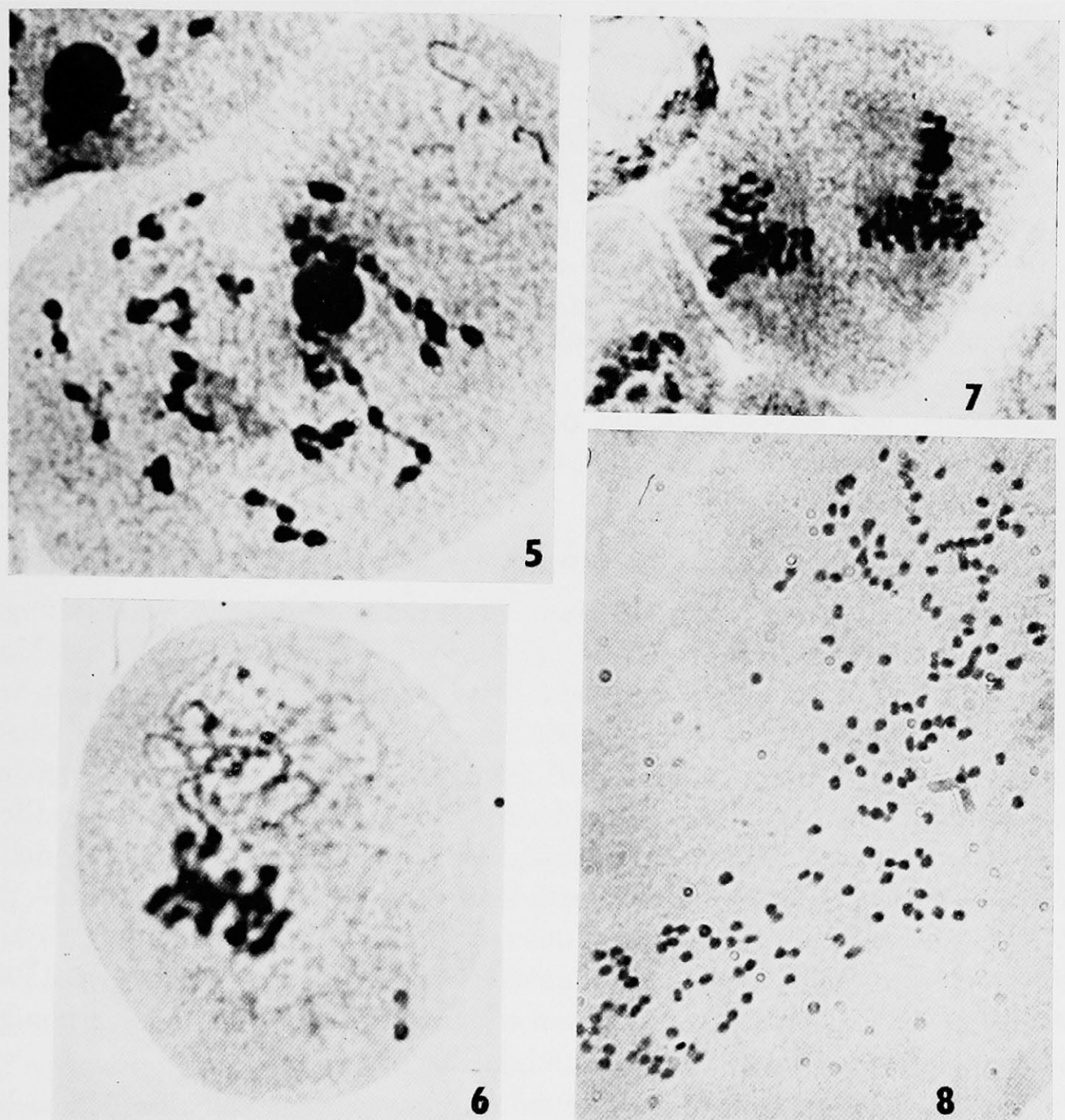

Abb. 5-8. 5, hypertetraploide Diakinese der tetraploiden Versuchspflanze Nr. 1412. 48 Chromosomen der Zelle $(=4 \mathrm{n})$ liegen im Kern in Form normaler Bi- und Multivalente vor, während die überzähligen Chromosomen in kaum spiralisierter Form an der Peripherie der Zelle liegen und nicht am meiotischen Stadienablauf teilnehmen (oben rechts). 6, 1 Metaphase einer Pollenmutterzelle der tetraploiden Pflanze Nr. 934. Die Chromosomenzahl der unteren Chromosomengruppe liegt in der Größenordnung von 2n, darüber schwach spiralisierte Chromosomen in pachytän-ähnlichem Zustand. 7, 2 tripolare Spindeln in der 2. Metaphase einer oktoploiden Pollenmutterzelle der Versuchspflanze Nr. 75. 8, Restitutionskernbildung in den späten Stadien der 2. meiotischen Teilung einer PMZ der okto.jloidenVers uchspflanze $\mathrm{Nr}$. 1. In der Zelle sind etwa 160 Chromosomen vorhanden $(168=14 \mathrm{n})$; Spindelbildung ist nicht nachweisbar.

auch während der prämeiotischen Mitosen ab und führen zu PMZ mit besonders hohen Polyploidiestufen.

Außer den beschriebenen Degenerationsvorgängen und Spindelanomalien 
wurde noch ein breites Spektrum der verschiedenartigsten meiotischen Störungen beobachtet, die im einzelnen nicht behandelt werden sollen. Es handelt sich hierbei u.a. um folgende UnregelmäBigkeiten:

- aneuploide, häufig hypoploide Chromosomenzahlen,

- Verteilungsstörungen, Bildung von Mikronuklei,

- Verklebungen, Brückenbildungen,

- Membranbildung nach Ablauf der 1. meiotischen Teilung,

- sehr hohe Gonenzahl innerhalb der PMZ,

- Überalterungserscheinungen und Stillstand der Meiosis in den frühesten Stadien der 1. Prophase,

- Anomalien im Spiralisationsgrad aller Chromosomen,

- Unregelmäßigkeiten im Formwechsel der Nukleolen,

- Bildung mehrkerniger PMZ,

- Auftreten von Riesenzellen mit dem vielfachen Volumen normaler PMZ,

- ungewöhnlich breite Stadienstreuung innerhalb der Anthere (Zygotän bis 2. Interphase).

3. Die Häufigkeit degenerativer Prozesse

Die in den vorherigen Abschnitten beschriebenen meiotischen Störungen sind in der Mehrzahl aller Fälle mit der Bildung nicht funktionsfähiger Keimzellen verbunden; es dürfte daher von Interesse sein, nähere Auskünfte über ihre Häufigkeit zu erhalten. Ein besonderes Charakteristikum dieser Vorgänge besteht darin, daß sie in bestimmten Antherenregionen gehüuft auftreten, während die PMZ benachbarter Regionen der gleichen Anthere eine völlig normale Meiosis durchlaufen. Exakte Zahlenangaben sind daher an die Auswertung vollständiger Antheren gebunden. Da die Anzahl der PMZ je Anthere bei tetraploiden Tomaten in der Größenordnung von 7000, bei oktoploiden Individuen noch immer in der Größenordnung von 15002000 liegt, ist ein derartiges Verfahren mit einem großen Aufwand verbunden und kann nicht auf breiter Basis durchgeführt werden.

Bei einer quantitativ ausgewerteten Anthere der tetraploiden Versuchspflanze Nr. 599 erreichte der Anteil kernloser PMZ einen Wert von 6\%, hinzu kommt ein etwas größerer Anteil von Zellen mit Degenerationserscheinungen. Hier wird also etwa ein Achtel aller PMZ schon aus diesem Grunde für die Keimzellenbildung ausfallen. Bei der ebenfalls tetraploiden Pflanze Nr. 934 wurden Antherenregionen mit je 500-600 PMZ quantitativ ausgewertet, die kaum eine normale Meiosis zeigten, während in den übrigen Zonen keine Störungen zu beobachten waren. Der gleiche Effekt trat bei anderen tetra- und oktoploiden Versuchspflanzen in Erscheinung und darf für unser Material verallgemeinert werden; er ist für die Interpretation unserer Befunde von Interesse (s.u.).

Für die übrigen Anomalien gelten die gleichen Gesetzmäßigkeiten. So wurden innerhalb eines kleinen Bereichs einer Anthere der tetraploiden 
Pflanze Nr. 934 mehrere hundert PMZ gefunden, bei denen die Spindelbildung unterblieben war; analoge Verhältnisse traten bei oktoploiden Pflanzen auf. Schließlich ist noch darauf hinzuweisen, da $B$ außer den pathologischen Veränderungen der polyploiden $\mathrm{PMZ}$ noch vielfältige Verteilungsstörungen der Chromosomen als Folge der Anwesenheit von Uni- und Multivalenten zu erwarten sind, die den Anteil fertiler Gonen weiter herabsetzen werden. Durch eine Summierung aller dieser Vorgänge ist mit einem hohen Gonenausfall zu rechnen, für den im einzelnen keine genauen Werte angegeben werden können. Er wird nicht nur von Blüte zu Blüte, sondern vornehmlich von Pflanze zu Pflanze stark variieren. Wir haben in unseren Colchicinversuchen neben tetraploiden Individuen mit sehr hohen Störungsraten auch solche mit auffallend präzis ablaufender Meiosis gefunden. Unser Versuchsmaterial setzte sich also-obwohl wir von reinen Linien ausgegangen sind-aus cytologisch stabilen und cytologisch labilen Organismen zusammen. Die Ursachen für ein derartig unterschiedliches karyologisches Verhalten sind noch nicht bekannt.

\section{Diskussion}

Teilungsanomalien, die jenen Vorgängen entsprechen, die im empirischen Teil der vorliegenden Arbeit behandelt worden sind, sind als Einzelvorgänge für die Pathologie der Mitose und Meiosis längst bekannt. Es sei darauf verzichtet, die große Zahl vergleichbarer Befunde zu zitieren, ich möchte vielmehr auf die Angaben bei Tischler (1951) sowie Tischler und Wulff (1953-63) verweisen. Das Charakteristikum unserer Befunde liegt darin, daß die ganze Vielfalt dieser Anomalien innerhalb der gleichen Pflanze, häufig sogar innerhalb der gleichen Ant he re nachweisbar ist

Auffallenderweise trat das breite Spektrum meiotischer Unregelmäßigkeiten nicht bei jeder Pflanze unseres polyploiden Versuchsmaterials in Erscheinung. Der größte Teil der tetraploiden Pflanzen zeigte vielmehr bei guten Fertilitätsverhältnissen die für autotetraploide Organismen allgemein bekannten relativ geringfügigen cytologischen Störungen, kann folglich als cytologisch stabil betrachtet werden. Nur bei wenigen tetraploiden und bei allen höherpolyploiden Individuen waren wesentlich stärkere Anomalien feststellbar. Interessanterweise traten hierbei zwei in ihrer Wirkung völlig verschiedenartige Phänomene gemeinsam in Erscheinung: neben vielfältigen pathologischen Kernveränderungen, die teils die Sistierung der Meiosis, zumindest aber die Ausbildung nicht funktionsfähiger Keimzellen zur Folge haben, fanden sich genomatische Regulierungsprozesse. Dieser Zusammenhang scheint von besonderem Interesse zu sein; er zeigt, daß unsere Befunde nicht als gehäuft auftretende meiotische Anomalien schlechthin aufzufassen sind, sie erfordern vielmehr eine andere Interpretation.

Es sei daran erinnert, daß die Degenerationserscheinungen vornehmlich 
in a neuploiden PMZ zu beobachten sind. Das meiotische Verhalten derartiger Zellen läßt sich folgendermaßen interpretieren : Durch prämeiotische UnregelmäBigkeiten sind aneuploide PMZ unterschiedlicher Valenz entstanden, die infolge ihrer unausgeglichenen genomatischen Verhältnisse eigentlich zu nicht funktionsfähigen Gonen führen müBten. Während des meiotischen Stadienablaufs werden jedoch Regulierungsmechanismen wirksam, die trotz der Aneuploidie eine genomatische Ordnung im Sinne der Bildung e up lo ider Kerne herbeiführen. Die überzähligen Chromosomen degenerieren. Durch eine Verbindung von Regulation und Degeneration wird also ein genomatisch ungeordneter Zustand in einen geordneten Zustand überführt. Die Degenerationsvorgänge können folglich nicht ausschließlich als pathologische und damit als negative Erscheinungen gewertet werden, sie erfüllen in unserem Material vielmehr eine spezifische Funktion: sie wirken in Verbindung mit Regulationsprozessen an der Realisierung eines neuen Gleichgewichtszustands innerhalb der Zelle mit und stellen in diesem Zusammenhang ein Positivum dar.

Uber die Ursachen der unterschiedlichen cytologischen Stabilität verschiedener Pflanzen der gleichen Polyploidiestufe können noch keine Aussagen gemacht werden. Sie liegen nicht auf genetischem Sektor, denn wir sind von reinen Linien ausgegangen. Vielleicht besteht eine Korrelation zur Colchicinmenge, die die Embryonen aufgenommen haben und die während der Ontogenese bis zu einem gewissen Grade physiologisch wirksam bleibt.

Es sei nunmehr noch versucht, die Ursachen der Degenerationserscheinungen $z u$ interpretieren. Es läßt sich generell feststellen, daß die meiotischen Unregelmäßigkeiten unseres Materials innerhalb der Anthere nicht zufallsgemä $B$ verteilt sind, sie treten vielmehr in bestimmten Antherenregionen gehäuft auf, während die übrigen $\mathrm{PMZ}$ eine störungsfreie Meiosis durchlaufen. Diese "Nesterbildung" kann in zweierlei Weise interpretiert werden. Zunächst wäre es denkbar, daß die Voraussetzungen für den später auftretenden Defekt bereits während des Ablaufs der prämeiotischen Mitosen in einer Archesporzelle induziert werden. Es wäre möglich, daß die Deszendenten der geschädigten Zelle die für den Defekt verantwortliche "Substanz" ebenfalls enthalten und über die Zellteilung hinweg an ihre Tochterzellen weitergeben. Wenn ein derartiger Vorgang in einem relativ späten Stadium der Antherenentwicklung induziert wird, ist eine Nesterbildung $z u$ erwarten. Sie kann jedoch auch auf andere Weise zustande kommen, die meiner Auffassung nach wahrscheinlicher ist. So wäre es möglich, daß bestimmte störende Einflüsse von aussen auf eine ganze Zellgruppe eines Pollensacks einwirken und da $B$ die PMZ der betreffenden Antherenregion durch die Realisierung übereinstimmender Anomalien auf diese Störung reagieren. Über die Natur derartiger Reize können noch keine Aussagen gemacht werden; es ist aber für beide Interpretationen sehr wahrscheinlich, daß die eigentlichen Ursachen nicht im Archespor, sondern im Tapetum zu suchen 
sind. So könnten lokale Störungen im Stoffwechsel dieses für die Ernährung der PMZ verantwortlichen Gewebes zur Abgabe von Substanzen führen, die von den Archespor- bzw. Pollenmutterzellen als Reiz empfunden werden und bestimmte Reaktionen auslösen. Da der gleiche Reiz einwirkt, wird auch die Reaktion gleichartig sein. Geringe morphologisch erkennbare Unterschiede in der Ausprägung bestimmter Störungstypen könnten auf Konzentrationsunterschiede des als Reiz fungierenden stofflichen Agens zurückzuführen sein. Mit Hilfe dieser Hypothese werden zahlreiche Befunde an unserem Material verständlich. Soweit degenerative Vorgänge mit bestimmten Regulierungsmechanismen korreliert sind, müssen wir die Ursachen der Degeneration allerdings in der Zelle selbst suchen.

\section{E. Summary}

From the seeds of diploid and tetraploid tomatoes germinated on colchicine agar a small number of tetraploid and octoploid plants was obtained. Some of them showed manifold meiotic irregularities in addition to the usual cytological disturbances of polyploids. In the present paper degeneration of chromosomes and spindle anomalies are described.

A direct correlation between regulative and degenerative processes could be observed in many aneuploid pollen mother cells of our polyploid material. A seperation of complete euploid chromosome complements took place while the supernumerary chromosomes of these cells degenerated. Thus, a genomatically balanced euploid final situation was formed starting from an unbalanced aneuploid initial situation.

Manifold spindle abnormalities some of them being again correlated with genomatic regulation cause atypical courses of meiosis.

The meiotic irregularities observed do not show a random distribution within the anther but they are accumulated in distinct areas. This "nestformation" is interpreted as a consequence of local metabolic disturbances in the tapetum tissue. The frequency of meiotic irregularities varies widely if we compare different plants of the same level of ploidy. Therefore, our material can be subdivided in cytologically stable plants showing a nearly normal meiosis and cytologically instable plants containing a wide spectrum of meiotic irregularities.

\section{Literatur}

Gottschalk, W. 1958a. Untersuchungen zum Problem der Herabregulierung der Chromosomenzahl in experimentell erzeugten polyploiden Pflanzen. Z. Vererbungslehre 89: $52-79$.

- 1958b. Uber Abregulierungsvorgänge bei künstlich hergestellten hoch polyploiden Pflanzen. 2. Vererbungslehre 89: 204-215.

- 1959. Die Bildung von Keimzellen mit euploiden Chromosomenzahlen aus triploiden und aneuploiden Pollenmutterzellen abregulierender polyploider Pflanzen. Z. Vererbungslehre 90: 198-214. 
- 1960. Meiotische und prämeiotische Abregulierung. Z. Bot. 48: 104-125.

- und Heide, N. 1960. Uber das Auftreten tripolarer Spindeln und mehrkerniger Pollenmutterzellen in abregulierenden polyploiden Pflanzen. Z. Vererbungslehre 91: $27-44$.

Swaminathan, M. S. 1950. Einige Verfahren für die Verwendung wilder Solanum-Arten zu Zuchtzwecken. Züchter 20: 358-360.

Tischler, G. 1951. Handbuch der Pflanzenanatomie, Bd. 2. Allgemeine Pflanzenkaryologie. 2. Hälfte: Kernteilung und Kernverschmelzung. Berlin.

- und Wulff, H. D. 1953-1963. Angewandte Pflanzenkaryologie. Berlin. 